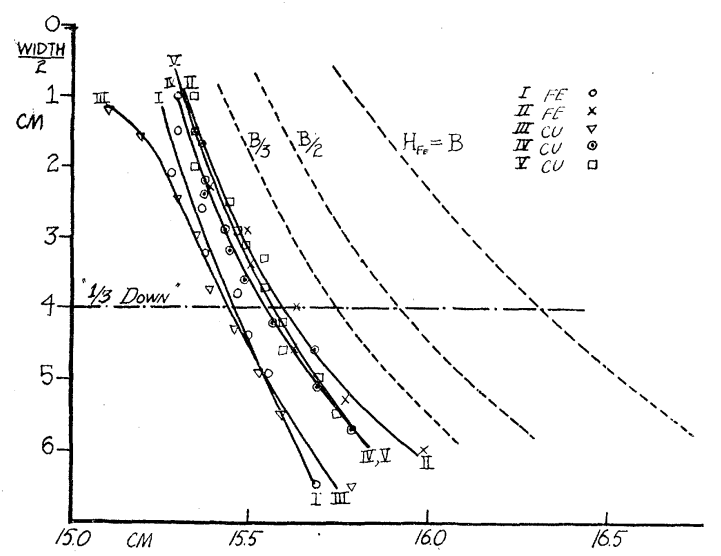

FIG. 1.

consistent with the findings of Mott-Smith, ${ }^{3}$ and Rossi, ${ }^{4}$ in conjunction with the later work of Curtiss. ${ }^{5}$ The data are also in accord with the view that the interior field is equal to the field in a spherical cavity, and not to that in the usually assumed, pill-box shaped cavity. In this case, the equation is $\mathrm{H}_{\mathrm{Fe}}=(2+\mu) \mathrm{H}_{\mathrm{a} \text { ir }} / 3$.

In the hope of evaluating $\mathrm{H}_{\mathrm{Fe}}$, the experiments are being continued, by using a piece of cobalt iron $(B=25,000)$ and a greater distance from iron to film. The expected shift could also be tripled, and the scattering reduced, by using the beta-rays of thorium $\mathrm{C}^{\prime \prime}$, a thicker sheet of iron, and a reversal of the induction. In this case, no focussing field would be necessary, since the iron sheet would filter out a nearly homogeneous beam of beta-rays of initial $\mathrm{H} \rho=10^{4}$

The author expresses with pleasure, his indebtedness to Dr. James Thompson for the loan of several radon tubes, and to Professors A. H. Compton and J. B. Hoag for suggestions and encouragement during the course of the experiment.

University of Chicago, January 16, 1934 .

${ }^{3}$ L. M. Mott-Smith, Phys. Rev. 39, 403 (1932).

${ }^{4}$ B. Rossi, Nature 128, 300 (1931).

${ }^{5}$ L. F. Curtiss, Bur. Standards J. Research 9, 815 (1932).

\title{
Disintegration of Beryllium by Deutons
}

We reported recently ${ }^{1}$ that neutrons were produced in large numbers when beryllium was bombarded with deutons having energies between 400,000 and 800,000 electron-volts. This has since been confirmed by Livingston, Henderson and Lawrence, ${ }^{2}$ by using deutons of considerably higher energy. In our early measurements of neutrons the ionization chamber was protected by a lead armor $5 \mathrm{~cm}$ thick, in order to minimize the effect of other types of radiation. Realizing that nuclear $\gamma$-radiation might be produced in the disintegration, we later made provision for reducing the thickness of lead in the direct path of the radiation to $\frac{1}{4}$ inch. With this arrangement we have measured the absorption of the beryllium radiation in lead and in paraffin, using in each case a lead and a paraffin lined ionization chamber. The paraffin lined chamber is more than twice as sensitive to neutrons as the lead lined chamber, and only 0.6 times as sensitive to $\gamma$-radiation as the lead lined chamber. Thus by comparing the absorption curves obtained with the two chambers, a mixture of neutrons and $\gamma$-rays can be to some extent analyzed.

Four absorption curves, obtained by using the two kinds of absorber and the two chambers, are shown in Fig. 1. Consider first the curves (II and IV) for the lead absorber, at thicknesses greater than $4 \mathrm{~cm}$. The slope is the same for the lead and the paraffin chambers, indicating that the radiation is of a single type, either entirely neutrons or entirely $\gamma$-rays. The large displacement of curve II (paraffin chamber) above curve IV (lead chamber) shows clearly that this radiation is neutrons. The slope of curves II and $\mathrm{IV}$, beyond $4 \mathrm{~cm}$ absorption is therefore taken to be the absorption coefficient for the neutrons in lead. At thicknesses of absorber less than $4 \mathrm{~cm}$, curve IV shows a steep rise, which clearly indicates the presence of a component of

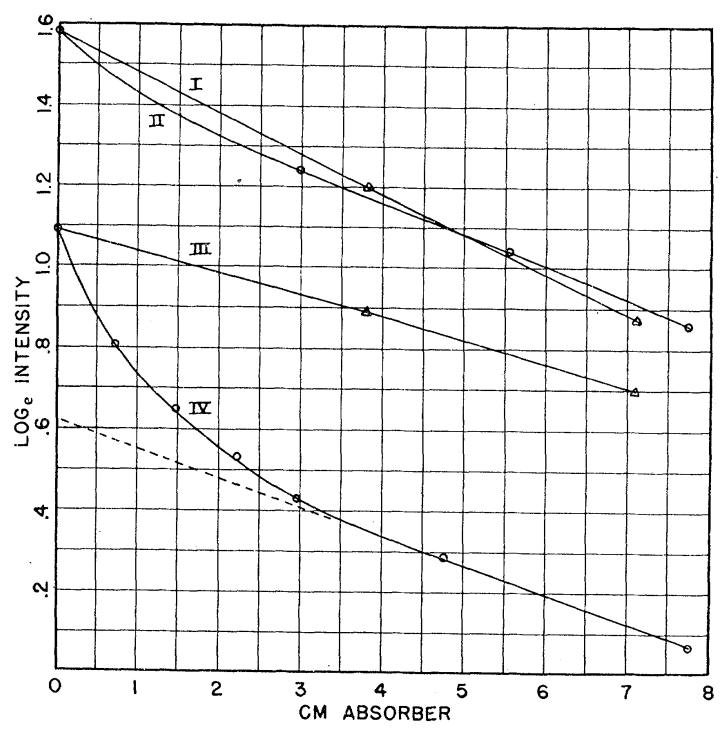

FIG. 1. Absorption of the beryllium radiation. I. Paraffin lined chamber, paraffin absorber; II. Paraffin lined chamber, lead absorber; III. Lead lined chamber, paraffin absorber; IV. Lead lined chamber, lead absorber.

radiation which is much more absorbable than the neutrons, and which is almost entirely screened out by $4 \mathrm{~cm}$ of lead. That this component is $\gamma$-radiation is indicated by the fact

${ }^{1}$ Crane, Lauritsen and Soltan, Phys. Rev. 44, 692 (1933).

${ }^{2}$ Livingston, Henderson and Lawrence, Phys. Rev. 44, 782 (1933). 
that it shows up very much more in the lead chamber curve than in the corresponding paraffin chamber curve. By extending the straight part of curve IV backward, as indicated by the dotted line in Fig. 1, we can determine the intensity contributed by neutrons alone, for absorbers less than $4 \mathrm{~cm}$. The difference between the total intensity and the intensity represented by the dotted line should then be just the intensity due to the $\gamma$-rays. Since all the curves are on a $\log$ scale, the intensity of $\gamma$-rays will be the difference between the antilogs of corresponding points on curve IV and the dotted line. The intensity of $\gamma$-rays thus obtained, as a function of thickness of absorber, is plotted on a log scale in Fig. 2. An absorption curve for the $\gamma$-rays from a radium source made under the same experimental conditions is shown for comparison. The $\gamma$-rays from beryllium appear to be monochromatic, and their absorption coefficient is the same as that of radium $\gamma$-rays after 1 or $2 \mathrm{~cm}$ lead filtration.* Since the strength of the radium source is known, the number of $\gamma$-rays emitted from beryllium can be calculated, and it turns out to be equal to the number of neutrons, to well within the accuracy with which we are able to determine the number of neutrons. It is therefore supposed that the neutrons and the $\gamma$-rays are produced in the same reaction, namely

$$
\mathrm{Be}^{9}+\mathrm{H}^{2} \rightarrow \mathrm{B}^{10}+n^{1}+\gamma .
$$

The above measurements were made at 800,000 volts and 2 microamperes deuton current, and under these conditions we find that the number of $\gamma$-rays produced is approximately equal to the number emitted by 0.15 milligram of radium. It is interesting to note that the $\gamma$-rays observed in

* A telegram from the authors informs me that the ordinate scale for the beryllium curve in Fig. 2 is wrong by a factor of 2. The gamma rays correspond therefore more nearly to 100 million volts than to the gamma rays from radium as stated.-EDITor.

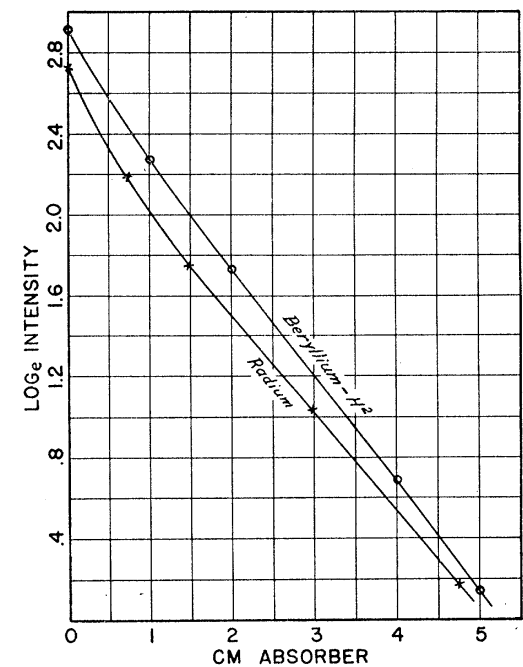

FIG. 2. Absorption curve of the beryllium $\gamma$-rays, with an absorption curve of radium $\gamma$-rays for comparison.*

the present experiment are, as closely as we can determine, of identical hardness with those produced in the disintegration of lithium by protons. ${ }^{3}$

We $w$ ish again to express our gratitude for support received from the Seeley W. Mudd Fund, which has made this, as our previous experiments, possible.

H. R. Crane

C. C. Lauritsen

Kellogg Radiation Laboratory, California Institute of Technology, January 17, 1934.

${ }^{3}$ Lauritsen and Crane, Phys. Rev. 45, 63 (1934). 